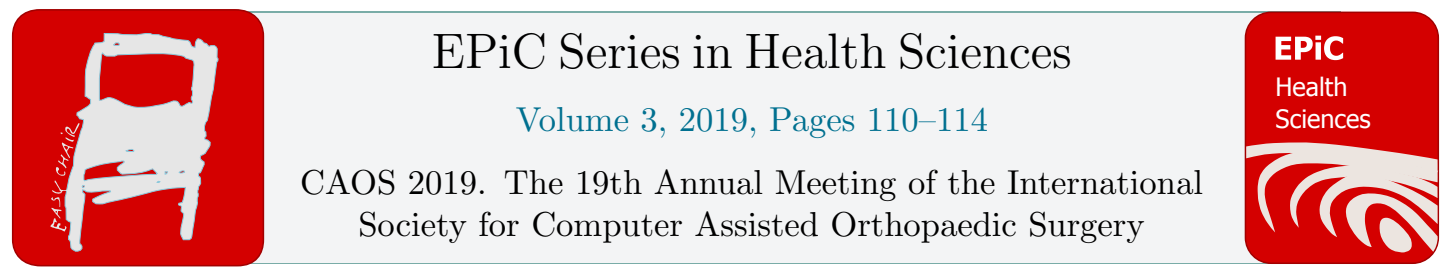

\title{
Towards a Commercial System for Intraoperative Manufacture of Patient-Specific Guides for Shoulder Arthroplasty
}

\author{
Alastair Darwood ${ }^{1}$, Simon Hurst ${ }^{1}$, Guillaume Villatte ${ }^{2}$, Ryan Fenton ${ }^{3}$, Fabio \\ Tatti $^{1}$, Hadi El-Daou ${ }^{1}$, Peter Reily ${ }^{1}$, Roger Emery ${ }^{1}$, Ferdinando. Rodriguez y \\ Baena $^{1}$ \\ 1. Imperial College, London
}

2. Centre Hospitalier Universitaire de Clermont-Ferrand, France

3. Desoutter Medical Ltd.

Corresponding author contact: Dr. Alastair Darwood, Imperial College, London, UK, SW7 2AZ

+447824668221 | alastairdarwood@,hotmail.com

\begin{abstract}
The accurate placement of orthopaedic implants according to a biomechanically derived preoperative plan is an important consideration in the long-term success of these interventions. Guidance technologies are widely described however, high cost, complex theatre integration, intraoperative inefficiency and functional limitations have prevented the widespread use. A novel, intraoperative mechatronics platform is presented, capable of the rapid, intraoperative manufacture of low-cost patient-specific guides. The device consists of a tableside robot with sterile drapes and some low cost, sterile disposable components. The robot comprises a 3D optical scanner, a three-axis sterile computer numerical control (CNC) drill and a two-axis receptacle into which the disposable consumables may be inserted. The sterile consumable comprises a region of rapidly setting moldable material and a clip allowing it to be reversibly attached to the tableside robot. In use, patient computed tomography (CT) imaging is obtained at any point prior to surgery and a surgical plan is created on associated software. This plan describes the axis and positioning of one or more guidewires which may, in turn, locate the prosthesis into position. Intraoperatively, osseous anatomy is exposed, and the sterile disposable is used to rapidly create a mould of the joint surface. Once set, the mould is inserted into the robot and an optical scan of the surface is taken followed by automatic surface registration, bringing the optical scan
\end{abstract}


Towards a Commercial System for Intraoperative Manufacture of Patient-Specific .. $\quad$ Darwood et al.

into the same coordinate frame of reference as the CT data and plan. The $\mathrm{CNC}$ drill is orientated such that the drill axis and position exactly matches the planned axis and position with respect to the moulded surface. A guide hole is drilled into the mould blank, which is removed from the robot and placed back into the patient with the moulded surface ensuring exact replacement. A wire is subsequently driven through the guide hole into the osseous anatomy in accordance with the preoperative plan. The guide blank may be slid off the wire and discarded allowing the procedure to continue in the normal manner. A Pre-clinical prototype robot was constructed and used to insert guide wires into a 12 cadaveric shoulder arthroplasty specimens according to a 'pre-operative' plan. A 'postoperative' CT scan was used to assess accuracy with respect to the 'pre-operative' plan. Initial average end to end accuracy was $1.2 \mathrm{~mm}$ with respect to planned vs achieved glenoid point of entry and $2.2 \mathrm{deg}$ version, $1.6 \mathrm{deg}$ inclination with respect to planned vs achieved wire angle. These data demonstrate accuracy within the bounds of relevant literature with a significant margin for potential improvement.

\section{Introduction}

The accurate placement of orthopaedic implants according to a biomechanically derived preoperative plan is an important consideration in the long-term success of these interventions ${ }^{1}$. Guidance technologies are widely described and comprise active or passive intra-operative robotics platforms, and three dimensional (3D) printed patient specific jigs $^{2}$. Despite improvements in placement accuracy, high cost, complex theatre integration, intraoperative inefficiency and functional limitations have prevented the widespread adoption of these technologies ${ }^{3}$.

A novel, precommercial intraoperative mechatronics platform is presented, which is capable of the rapid, intraoperative manufacture of low-cost patient-specific guides ${ }^{4}$. The device consists of a tableside robot (Fig. 1, A) with sterile drapes (Fig. 1, B) and some low cost, sterile disposable components. The robot comprises a 3D optical scanner (Fig. 1, C), a three-axis sterile computer numerical control (CNC) drill (Fig. 1, D) and a two-axis receptacle into which the disposable consumables may be inserted (Fig. 2, A). The sterile consumable comprises a region of rapidly setting moldable material (Fig. 1, E) and a clip (Fig. 1, F) allowing it to be reversibly attached to the tableside robot.

In use, patient computed tomography (CT) imaging is obtained at any point prior to surgery and a surgical plan is created on associated software (Fig. 2, B). This plan describes the axis and positioning of one or more guidewires which may, in turn, locate the prosthesis into position. Intraoperatively, osseous anatomy is exposed, and the sterile disposable is used to rapidly create a mould of the joint surface (Fig. 1, G). Once set, the mould is inserted into the robot and an optical scan of the surface is taken followed by automatic surface registration, bringing the optical scan into the same coordinate frame of reference as the CT data and plan (Fig. 2, C). The CNC drill is orientated such that the drill axis and position exactly matches the planned axis and position with respect to the moulded surface. A guide hole is drilled into the mould blank (Fig. 2, D), which is then removed from the robot and placed back into the patient with the moulded surface ensuring exact replacement. A wire is subsequently driven through the guide hole into the osseous anatomy in accordance with the preoperative plan (Fig. 2, E). The guide blank may be slid off the wire and discarded allowing the procedure to continue in the normal manner. 
Towards a Commercial System for Intraoperative Manufacture of Patient-Specific .. $\quad$ Darwood et al.

\section{Objectives}

We performed a cadaveric trial to asses prototype function in the placement of a guide wire into a preoperatively planned position within the glenoid cavity during a simulated total shoulder arthroplasty procedure.

\section{Methods}

A prototype device was constructed along with planning software and sterile consumables (Fig. 2, A). A kinematic calibration and assessment of the device was carried out to identify the mechanical accuracy of the system. 12 cadaveric shoulder specimens were obtained (sex, age and side randomized) and a preoperative CT scan performed on each specimen. A senior orthopaedic surgeon planned the ideal wire placement in each specimen and exposed the glenoid cavity via a conventional deltopectoral approach. The device and sterile disposables were used to guide the placement of guide wires into the glenoid cavity. Following wire insertion, a postoperative CT scan was obtained for each specimen. The pre- and postoperative scans were then segmented and coregistered to allow the planned vs achieved wire position to be computed.

\section{Results}

The overall kinematic accuracy of the prototype was $0.50 \pm 0.26 \mathrm{~mm}$ in terms of translational positioning and $0.59 \pm 0.30^{\circ}$ angular accuracy. The cadaveric trial results (MAD) show overall end to end placement accuracy of $1.18 \mathrm{~mm}(0.22)$ with respect to planned vs achieved glenoid point of entry and $2.15 \mathrm{deg}(0.96)$ version, $1.63 \mathrm{deg}$ (0.59) inclination with respect to planned vs achieved wire angle. ( $\mathrm{p}$ values, $0.002,0.002$ and 0.003 respectively).

\section{Discussion}

This technology and cadaveric study demonstrate the ability to rapidly produce functional guides in accordance with a pre-operative plan. Guide wires were accurately and rapidly inserted, whilst the consumables and the compact, intraoperative platform may result in possible cost reductions and efficiency improvements. The novel registration method making use of patient anatomy found at the time of surgery may provide additional accuracy improvements and added functionality in cases that are traditionally challenging to conventional 3D printed orthopaedic guides. Registration of the moulded surface with the CT data could leverage optimizations in the algorithm's design, such as filtering, weighing and local minima suppression, to ensure a reliable and repeatable replacement fit into the surgical site. Glenoid component malpositioning has been shown to be a major contributing factor to early failure and poor prosthesis function, thus guidance technologies may play an important role in improving patient outcomes ${ }^{5}$. 
Towards a Commercial System for Intraoperative Manufacture of Patient-Specific .. $\quad$ Darwood et al.

This cadaveric trial demonstrates that the intraoperative manufacture of patient-specific guides is viable and can yield results capable of surpassing traditional PSIs ${ }^{6}$. Further work is planned on other joint areas such as the hip and knee with a first-in-man trial planned for 2020.

\section{References}

1. Hopkins, A. R., Hansen, U. N., Amis, A. A. \& Emery, R. The effects of glenoid component alignment variations on cement mantle stresses in total shoulder arthroplasty. J. Shoulder Elbow Surg. 13, 668-75 (2004).

2. Malik, H. H. et al. Three-dimensional printing in surgery: a review of current surgical applications. $J$. Surg. Res. 199, 512-522 (2015).

3. Beringer, D. C., Patel, J. J. \& Bozic, K. J. An overview of economic issues in computer-assisted total joint arthroplasty. Clin. Orthop. Relat. Res. 463, 26-30 (2007).

4. Darwood, A. Method and Apparatus for Use in the Production of a Surgical Guide. US Pat. Number: US20160342766 (2013).

5. Gregory, T. M. et al. Accuracy of Glenoid Component Placement in Total Shoulder Arthroplasty and Its Effect on Clinical and Radiological Outcome in a Retrospective, Longitudinal, Monocentric Open Study. PLoS One 8, e75791 (2013).

6. Villatte, G. et al. Use of Patient-Specific Instrumentation (PSI) for glenoid component positioning in shoulder arthroplasty. A systematic review and meta-analysis. PLoS One 13, e0201759 (2018).

\section{Acknowledgements:}

The financial support of De Soutter Medical ltd., who commissioned the work and produced the precommercial prototype described in this study is gratefully acknowledged. The technology behind the intraoperative manufacture of patient-specific guides is patented (US2016342766).

\section{Figures:}


Towards a Commercial System for Intraoperative Manufacture of Patient-Specific .. $\quad$ Darwood et al.
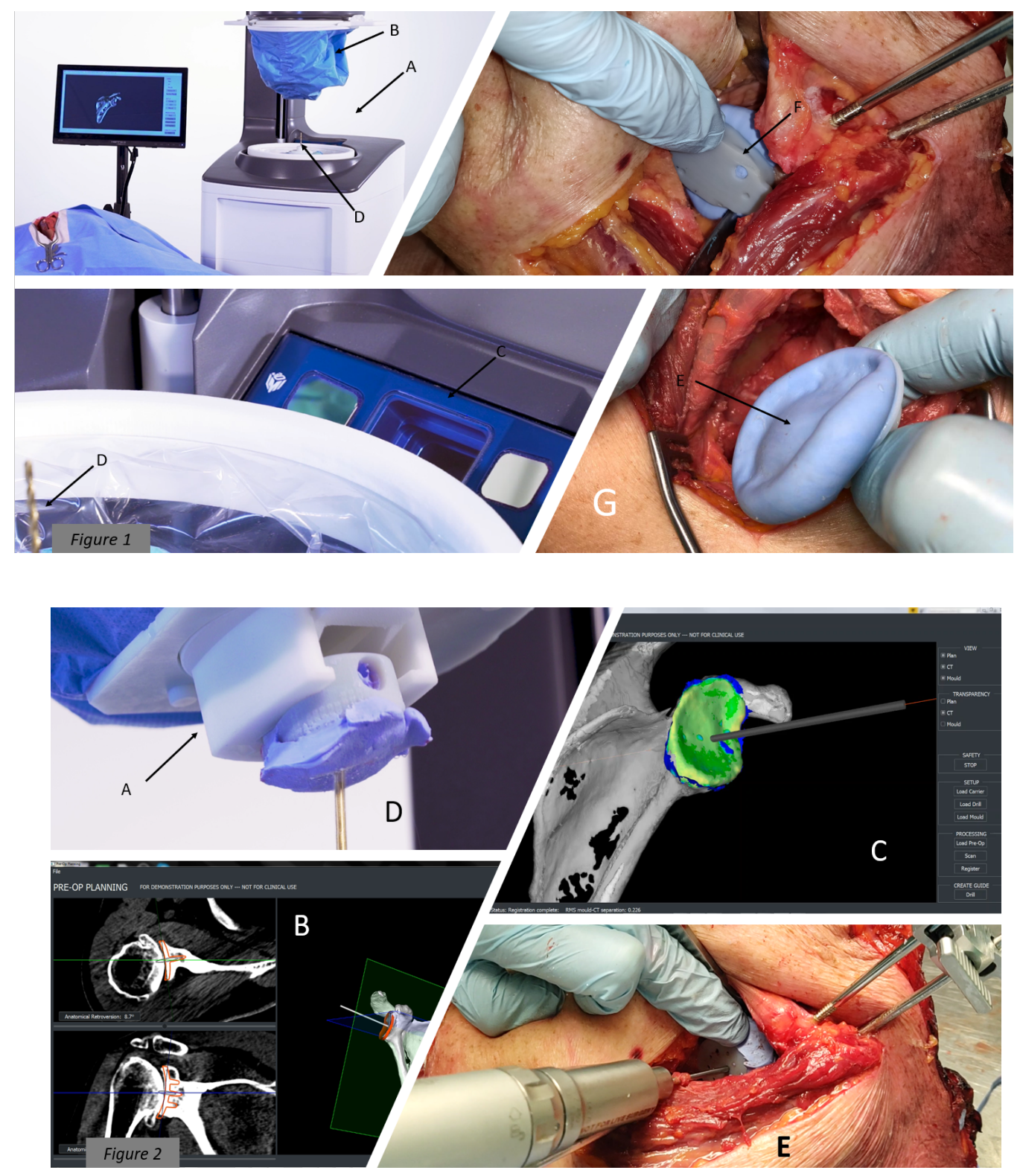